\title{
SOME GRID REFINEMENT SCHEMES FOR HYPERBOLIC EQUATIONS WITH PIECEWISE CONSTANT COEFFICIENTS
}

\author{
T. LIN, J. SOCHACKI, R. EWING, AND J. GEORGE
}

\begin{abstract}
Discontinuities in the coefficients of hyperbolic equations occur both naturally and artificially and must be treated in numerical schemes. Schemes for handling these discontinuities are derived. An interesting stability result is derived and the schemes are shown to be exact under certain restrictions.
\end{abstract}

\section{INTRODUCTION}

In this paper, we consider finite difference approximations of the following hyperbolic partial differential equations:

$$
\begin{gathered}
A(x) U_{t}(x, t)=(B(x) U)_{x}, \quad(x, t) \in D, \\
U(x, 0)=U_{0}(x),
\end{gathered}
$$

where $D=(-\infty, \infty) \times(0, \infty)$, and $A$ and $B$ are piecewise constant 'matrices' which are discontinuous at points $x=d_{1}, \ldots, d_{m}$. The $d_{i}$ 's are called the physical interfaces of the above hyperbolic systems. Usually, according to the physical properties of the process described by this class of hyperbolic equations, the solutions are required to satisfy the following conditions at the physical interfaces:

$$
B\left(d_{i}-\right) U\left(d_{i}-, t\right)=B\left(d_{i}+\right) U\left(d_{i}+, t\right), \quad t>0, i=1, \ldots, m .
$$

Two examples of these equations occur in geophysics and elasticity. The linearized acoustic wave equations are used in geophysics; in this context, the physical interfaces are the boundaries of geological structures occurring in the earth. The linearized elastic equations can be interpreted as the displacements of a "string" made up of materials of different composition connected together.

The example from acoustics is

$$
p_{t}+c^{2}(\rho v)_{x}=0, \quad v_{t}+\frac{1}{\rho} p_{x}=0
$$

Received May 2, 1989; revised April 19, 1990.

1980 Mathematics Subject Classification (1985 Revision). Primary 35L20, 35L50, 39A11, 65N 10.

Research partially supported by NSF Grant No. DMS-8712021, Office of Naval Research Contract No. 0014-88-K-0370, the Pittsburgh and Minnesota Supercomputer Centers, and the Institute for Scientific Computation at the University of Wyoming through NSF EPSCoR Grant RII-861 4115 . 
where $A=\left(\begin{array}{cc}1 / c^{2} & 0 \\ 0 & \rho\end{array}\right), B=-\left(\begin{array}{ll}0 & \rho \\ 1 & 0\end{array}\right), \rho$ is the density, $c$ is the speed of sound, $p$ is the pressure, and $v$ is the velocity. $p$ and $\rho v$ are required to be continuous at the interfaces. The elasticity example is

$$
\rho(x) V_{t t}=\frac{\partial}{\partial x}\left(\mu(x) V_{x}\right)
$$

where $V$ and $\mu V_{x}$ are required to be continuous at the interfaces. If we let $v=V_{t}$ and $w=\mu V_{x}$, we obtain

$$
\rho v_{t}=w_{x}, \quad \frac{1}{\mu} w_{t}=v_{x},
$$

and $A=\left(\begin{array}{cc}\rho & 0 \\ 0 & 1 / \mu\end{array}\right), B=\left(\begin{array}{ll}0 & 1 \\ 1 & 0\end{array}\right)$. Here, $\mu$ is the modulus of elasticity and $c^{2}=\mu / \rho$ is the speed of propagation of waves.

In finding approximate solutions to the above hyperbolic equations by finite difference schemes, the main difficulty occurs at the interfaces. The discontinuities of the coefficients at the interfaces prohibit us from using simple finite difference schemes to approximate the PDE within the usual order of accuracy. On the other hand, complex higher-order approximation schemes are usually more expensive and, very often, will result in instability. Since hyperbolic equations model wave propagation, their finite difference approximations must also have propagation properties. Even though we have many accurate finite difference approximation schemes for interior points, $x \neq d_{i}, i=1, \ldots, m$, the error generated at the interfaces may propagate out and detrimentally impact the computational results.

Another difficulty in the application of finite difference schemes in large-scale modeling is that we need very fine grid sizes to resolve the dramatically changing physics in some area of the domain, but we cannot afford to use such fine grids on the whole computational domain. One example is in seismic modeling, where the induced sources near the earth's surface generate high-frequency waves. If we want to compute these high-frequency waves accurately, we must use small grid sizes; however, using small grids in the whole modeling process will be too expensive, since the computational domains are usually very large. On the other hand, at locations far away from the source, where the high-frequency waves die out, we can model the physics accurately without using very fine grid sizes. Thus, in order to simulate the physics efficiently as well as accurately, we want to find schemes which use different grid sizes in different regions of the computational domains. This requirement introduces another difficulty: the numerical interfaces. In this paper, we want to discuss how to use the physical properties of hyperbolic equations to develop more accurate finite difference schemes that can handle both real and artificial interfaces.

Finite difference schemes for wave propagation problems containing physical and numerical interfaces have received significant attention in the literature. Brown [3] considered the wave propagation problem with piecewise constant coefficients and constant space and time step sizes. Vichnevetsky [13] discussed 
a scheme for (1.1) in the scalar case and analyzed the reflection from the physical and numerical interfaces. Trefethen [10] analyzed the 'GKS' stabilities [7] of some difference approximations to (1.1) with multiple interfaces. Berger [1], Oliger [2], Browning, Kreiss, and Oliger [4], and Ciment [5] discussed difference approximations to (1.1) with numerical interfaces. Sundström [9] addressed (1.1) for problems related to geophysics and noted that variable grid size could improve the accuracy of the finite difference solution to (1.1). De Moura [6] also considered a continuous variable grid scheme for the scalar case of (1.1) in dealing with weather prediction. Starius [8] derived some difference schemes for hyperbolic systems with variable grid size and proved their $l_{2}$ stability.

In this paper, we deal with the interfaces by using integral identities and characteristics. Since both ideas have physical motivations, they enable us to obtain schemes that can handle both types of interfaces satisfactorily. They are not only accurate enough (their local truncation errors are second-order on the whole computational domain), but they are also stable and can represent the transmitted and reflected waves very well.

To simplify the notation, we concentrate on the discussion of the following model problem with one physical and numerical interface at $x=0$ only:

$$
\begin{gathered}
a_{+} u_{t}(x, t)=b_{+} u_{x}(x, t), \quad x>0, t>0, \\
a_{-} u_{t}(x, t)=b_{-} u_{x}(x, t), \quad x<0, t>0, \\
u(x, 0)=f(x) .
\end{gathered}
$$

We impose the physical interface condition

$$
b_{-} u(0-, t)=b_{+} u(0+, t), \quad t>0 .
$$

The schemes derived for this model problem can be easily extended to more general problems with multiple physical and numerical interfaces, in which these two kinds of interfaces may be located at different places in the computational domain. Also, the ideas for deriving the schemes for the model problem can be applied to the second-order acoustic wave equation, since the second-order equation can be decomposed into a first-order equation system. The outline of the paper is as follows: In $\S 2$ we derive several schemes for the model problem and discuss their accuracy and extensions to more general and complex problems. In $\S 3$ we prove some corresponding stability results for the schemes derived in $\S 2$. In $\S 4$ we supply several numerical experiments for the schemes derived in this paper.

\section{Finite DiffERENCE SCHEMES}

We first introduce some notation. Let $h_{-}$and $h_{+}$denote the step sizes in the $x$ variable on each side of the interface $x=0$, let $k$ denote the step size in the $t$ variable, $t^{n}=n k, n=0,1,2, \ldots$, and let $x_{j}=j h_{\operatorname{sign}(j)}$, 
$j=0, \pm 1, \pm 2, \pm 3, \ldots$. Let $u_{j}^{n}$ and $v_{j}^{n}$ denote the finite difference approximations of $u\left(x_{j}, t^{n}\right)$ and $v\left(x_{j}, t^{n}\right)$, with $u_{0-}^{n}$ and $u_{0+}^{n}$ denoting the approximations of $u\left(0-, t^{n}\right)$ and $u\left(0+, t^{n}\right)$ at $x=0$. We also let $a_{j}=a\left(x_{j}\right)$ and $b_{j}=b\left(x_{j}\right)$.

The first scheme for the model problem is based on an integral identity satisfied by the hyperbolic equation at the interface. To obtain this integral identity, we integrate the partial differential equation (1.4) from $-h_{-} / 2$ to $h_{+} / 2$,

$$
\int_{-h_{-} / 2}^{h_{+} / 2} a(x) u_{t}(x, t) d x=\int_{-h_{-} / 2}^{h_{+} / 2}(b(x) u(x, t))_{x} d x .
$$

Since $b(x) u(x, t)$ is continuous across the interface, the right-hand side of the above identity satisfies

$$
\int_{-h_{-} / 2}^{h_{+} / 2}(b(x) u(x, t))_{x} d x=b_{+} u\left(\frac{h_{+}}{2}, t\right)-b_{-} u\left(-\frac{h_{-}}{2}, t\right) .
$$

Then, the integral identity has the form

$$
\begin{gathered}
a_{-} \int_{-h_{-} / 2}^{0} u_{t}(x, t) d x+a_{+} \int_{0}^{h_{+} / 2} u_{t}(x, t) d x \\
=b_{+} u\left(\frac{h_{+}}{2}, t\right)-b_{-} u\left(-\frac{h_{-}}{2}, t\right) .
\end{gathered}
$$

Now, we approximate the above identity term by term. For the first term on the right-hand side, we easily see that

$$
b_{+} u\left(\frac{h_{+}}{2}, t\right) \approx b_{+} \frac{u\left(x_{1}, t\right)+u(0+, t)}{2},
$$

and similarly,

$$
b_{-} u\left(-\frac{h_{-}}{2}, t\right) \approx b_{-} \frac{u\left(x_{-1}, t\right)+u(0-, t)}{2} .
$$

Based on the trapezoidal rule of integration, we have the following approximation for the second term on the left-hand side of (2.3):

$$
\begin{aligned}
& a_{+} \int_{0}^{h_{+} / 2} u_{t}(x, t) d x \\
& \approx a_{+} \frac{h_{+}}{4}\left(u_{t}\left(\frac{h_{+}}{2}, t\right)+u_{t}(0+, t)\right) \\
& \approx a_{+} \frac{h_{+}}{4}\left(\frac{u_{t}\left(x_{1}, t\right)+u_{t}(0+, t)}{2}+u_{t}(0+, t)\right) \\
& =a_{+} \frac{h_{+}}{8}\left(u_{t}\left(x_{1}, t\right)+3 u_{t}(0+, t)\right) \\
& \approx a_{+} \frac{h_{+}}{8}\left(\frac{u\left(x_{1}, t+k\right)-u\left(x_{1}, t-k\right)}{2 k}\right) . \\
& \left.+3 \frac{u(0+, t+k)-u(0+, t-k)}{2 k}\right) .
\end{aligned}
$$


Similarly, for the first term on the left-hand side of (2.3), we have

$$
\begin{aligned}
a_{-} \int_{-h_{-} / 2}^{0} u_{t}(x, t) d x \approx a_{-} \frac{h_{-}}{8} & \left(\frac{u\left(x_{-1}, t+k\right)-u\left(x_{-1}, t-k\right)}{2 k}\right. \\
& \left.+3 \frac{u(0-, t+k)-u(0-, t-k)}{2 k}\right) .
\end{aligned}
$$

Combining (2.2)-(2.7) yields

$$
\begin{aligned}
\frac{1}{2}\left[b_{+}\left(u_{1}^{n}+u_{0+}^{n}\right)-b_{-}\left(u_{-1}^{n}+u_{0-}^{n}\right)\right] \\
=\frac{a_{+} h_{+}}{16 k}\left[u_{1}^{n+1}-u_{1}^{n-1}+3\left(u_{0+}^{n+1}-u_{0+}^{n-1}\right)\right] \\
\quad+\frac{a_{-} h_{-}}{16 k}\left[u_{-1}^{n+1}-u_{-1}^{n-1}+3\left(u_{0-}^{n+1}-u_{0-}^{n-1}\right)\right],
\end{aligned}
$$

which is a second-order approximation of (1.4). For the finite difference approximation at the grid points not on the interface, we have many well-known schemes. For example, we use the following Leap Frog (LF) scheme:

$$
a_{\operatorname{sign}(j)} \frac{u_{j}^{n+1}-u_{j}^{n-1}}{2 k}=b_{\operatorname{sign}(j)} \frac{u_{j+1}^{n}-u_{j-1}^{n}}{2 h_{\operatorname{sign}(j)}}, \quad j \neq 0,
$$

which is a second-order approximation of the model equation. Since we use a \pm sign to indicate the grid function on the left- and right-hand sides of the interface, we need the following interface condition to complete the scheme:

$$
b_{-} u_{0-}^{n+1}=b_{+} u_{0+}^{n+1} \text {. }
$$

In summary, the finite difference scheme based on the integral identity for the model problem is the following

Scheme I.

$$
\begin{gathered}
a_{\operatorname{sign}(j)} \frac{u_{j}^{n+1}-u_{j}^{n-1}}{2 k}=b_{\operatorname{sign}(j)} \frac{u_{j+1}^{n}-u_{j-1}^{n}}{2 h_{\operatorname{sign}(j)}}, \quad j \neq 0, \\
b_{-} u_{0-}^{n+1}=b_{+} u_{0+}^{n+1}, \\
\frac{1}{2}\left(b_{+} u_{1}^{n}-b_{-} u_{-1}^{n}\right)=\frac{a_{+} h_{+}}{16 k}\left[u_{1}^{n+1}-u_{1}^{n-1}+3\left(u_{0+}^{n+1}-u_{0+}^{n-1}\right)\right] \\
+\frac{a_{-} h_{-}\left[u_{-1}^{n+1}-u_{-1}^{n-1}+3\left(u_{0-}^{n+1}-u_{0-}^{n-1}\right)\right] .}{16 k}
\end{gathered}
$$

This scheme is a semi-implicit scheme. To compute $u_{0 \pm}^{n+1}$, we compute $u_{j}^{n+1}$, $j \neq 0$, by equation (2.8); then we use equations (2.9) and (2.10) to compute $u_{0-}^{n+1}$ and $u_{0+}^{n+1}$. 
Remark 1. We can also approximate the terms on the left-hand side of $(2.3)$ as

$$
\begin{gathered}
a_{-} \int_{-h_{-} / 2}^{0} u_{t}(x, t) d x+a_{+} \int_{0}^{h_{+} / 2} u_{t}(x, t) d x \\
\approx a_{-} u_{t}(0-, t) \frac{h_{-}}{2}+a_{+} u_{t}(0+, t) \frac{h_{+}}{2} \\
\approx \\
\frac{a_{-} h_{-}}{4 k}(u(0-, t+k)-u(0-, t-k)) \\
\quad+\frac{a_{+} h_{+}}{4 k}(u(0+, t+k)-u(0+, t-k)) .
\end{gathered}
$$

Combining this with (2.8) and (2.9) results in the following interesting scheme for the model problem:

$$
\begin{gathered}
a_{\operatorname{sign}(j)} \frac{u_{j}^{n+1}-u_{j}^{n-1}}{2 k}=b_{\operatorname{sign}(j)} \frac{u_{j+1}^{n}-u_{j-1}^{n}}{2 h_{\operatorname{sign}(j)}}, \quad j \neq 0, \\
b_{-} u_{0-}^{n+1}=b_{+} u_{0+}^{n+1}, \\
\frac{1}{2}\left(b_{+} u_{1}^{n}-b_{-} u_{-1}^{n}\right)=\frac{a_{+} h_{+}}{4 k}\left(u_{0+}^{n+1}-u_{0+}^{n-1}\right)+\frac{a_{-} h_{-}}{4 k}\left(u_{0-}^{n+1}-u_{0-}^{n-1}\right) .
\end{gathered}
$$

Obviously, this scheme is less accurate than Scheme I from the point of view of local truncation error; however, this scheme gives the exact solution at the grid points if the initial function $f(x)$ has compact support in the region $x \geq 0$ and $\lambda_{ \pm}=1$, where

$$
\lambda_{ \pm}=\frac{b_{ \pm}}{a_{ \pm}} \frac{k}{h_{ \pm}} .
$$

In fact, for this case, the analytic solution of the model problem is

$$
u(x, t)= \begin{cases}\frac{b_{+}}{b_{-}} f\left(\frac{c_{+}}{c_{-}}\left(x+c_{-} t\right)\right) & \text { for } x<0, \\ f\left(x+c_{+} t\right) & \text { for } x>0,\end{cases}
$$

and if $\lambda_{ \pm}=1$, the values of this function at grid points become

$$
u\left(j h_{\mathrm{sign}(j)}, n k\right)= \begin{cases}\frac{b_{+}}{b_{-}} f\left((j+n) h_{+}\right), & j<0, \\ f\left((j+n) h_{+}\right), & j>0,\end{cases}
$$

where $c_{ \pm}=b_{ \pm} / a_{ \pm}$. Then, by substituting (2.16) into (2.12)-(2.14), we can easily show that the values of $u(x, t)$ at the grid points satisfy the difference equation. This means that the solution to the difference equation gives the analytic solution to the partial differential equation for any number of physical interfaces if we keep $\lambda=1$ for all grid points. Unfortunately, it is very hard to see how we can extend this scheme to more general cases, such as variable coefficients and/or higher-dimensional spaces, so that this property can still be preserved. 
We now make a change of variables, which is not necessary, but will make the proofs for the stability results easier. Let

$$
v(x, t)=b_{\operatorname{sign}(x)} u(x, t) .
$$

Then $v(x, t)$ is required to be continuous at the physical interface and satisfies the following equations:

$$
\begin{gathered}
a_{\operatorname{sign}(x)} v_{t}(x, t)=b_{\operatorname{sign}(x)} v_{x}(x, t), \quad x \neq 0, t>0, \\
v(x, 0)=b_{\operatorname{sign}(x)} f(x), \quad x \neq 0 .
\end{gathered}
$$

We call these equations the modified model equation. Since the modified model equation is equivalent to the model equation, the two finite difference schemes derived below are for this equation only. This time, we use the idea of characteristics to treat the interface. The characteristic segment for (2.18) passing through $\left(x_{0}, t^{n+1}\right)$ on the right-hand side of the interface is

$$
\left(x-x_{0}\right)+\frac{b_{+}}{a_{+}}\left(t-t^{n+1}\right)=0 .
$$

Since this segment lies completely on the right-hand side of the interface for $t<t^{n+1}$, the property of the characteristic segment suggests that we should be able to compute $v_{0+}^{n+1}$ from $v_{0+}^{n}$ and $v_{j}^{n}, v_{j}^{n+1}$, for $j>0$. Physically, this means that, since the wave described by the modified model equation is left-going, the displacements of the wave on the left-hand side of the interface have no influence on the displacements of the wave at the interface. In fact, integrating equation (2.18) over the volume element

$$
0 \leq x \leq \frac{h_{+}}{2}, \quad t^{n} \leq t \leq t^{n+1},
$$

gives

$$
\begin{aligned}
& a_{+} \int_{0}^{h_{+} / 2}\left(v\left(x, t^{n+1}\right)-v\left(x, t^{n}\right)\right) d x \\
& \quad=b_{+} \int_{t^{n}}^{t^{n+1}}\left(v\left(\frac{h_{+}}{2}, t\right)-v(0, t)\right) d t .
\end{aligned}
$$

Expanding the terms in these integrals gives

$$
\begin{aligned}
& v\left(x, t^{n+1}\right)-v\left(x, t^{n}\right) \\
& \quad \approx v\left(0, t^{n+1}\right)-v\left(0, t^{n}\right)+\left(v_{x}\left(0, t^{n+1}\right)-v_{x}\left(0, t^{n}\right)\right) x \\
& \quad \approx v\left(0, t^{n+1}\right)-v\left(0, t^{n}\right)+v_{t x}\left(0, t^{n}\right) k x
\end{aligned}
$$


for $0 \leq x \leq h_{+}$, and

$$
\begin{aligned}
& v\left(\frac{h_{+}}{2}, t\right)-v(0, t) \\
& \approx v\left(\frac{h_{+}}{2}, t^{n}\right)-v\left(0, t^{n}\right)+\left(v_{t}\left(\frac{h_{+}}{2}, t^{n}\right)-v_{t}\left(0, t^{n}\right)\right)\left(t-t^{n}\right) \\
& \approx v_{x}\left(\frac{h_{+}}{2}, t^{n}\right) \frac{h_{+}}{2}-v_{x x}\left(\frac{h_{+}}{2}, t^{n}\right) \frac{h_{+}^{2}}{8} \\
& \quad+v_{t x}\left(\frac{h_{+}}{2}, t^{n}\right) \frac{h_{+}\left(t-t^{n}\right)}{2} \\
& \approx v_{x}\left(\frac{h_{+}}{2}, t^{n}\right) \frac{h_{+}}{2}-\frac{a_{+}}{b_{+}} v_{x t}\left(\frac{h_{+}}{2}, t^{n}\right) \frac{h_{+}^{2}}{8} \\
& \quad+v_{t x}\left(\frac{h_{+}}{2}, t^{n}\right) \frac{h_{+}\left(t-t^{n}\right)}{2}
\end{aligned}
$$

for $t^{n} \leq t \leq t^{n+1}$. The corresponding approximation for equation (2.21) is

$$
\begin{aligned}
& a_{+}\left(v\left(0, t^{n+1}\right)-v\left(0, t^{n}\right)\right) \frac{h_{+}}{2}+a_{+}\left(v\left(h_{+}, t^{n+1}\right)-v\left(h_{+}, t^{n}\right)\right. \\
& \left.-v\left(0, t^{n+1}\right)+v\left(0, t^{n}\right)\right) \frac{h_{+}}{8} \\
& \approx a_{+} \int_{0}^{h_{+} / 2}\left[v\left(0, t^{n+1}\right)-v\left(0, t^{n}\right)+v_{x t}\left(0, t^{n}\right) k x\right] d x+O\left(h_{+}^{2}+k^{2}\right) \\
& \approx b_{+} \int_{t^{n}}^{t^{n+1}}\left[v_{x}\left(\frac{h_{+}}{2}, t^{n}\right) \frac{h_{+}}{2}-\frac{a_{+}}{b_{+}} v_{x t}\left(\frac{h_{+}}{2}, t^{n}\right) \frac{h_{+}^{2}}{8}+v_{x t}\left(\frac{h_{+}}{2}, t^{n}\right) \frac{h_{+}\left(t-t^{n}\right)}{2}\right] d t \\
& \approx b_{+}\left(v\left(h_{+}, t^{n}\right)-v\left(0, t^{n}\right)\right) \frac{k}{2} \\
& \quad-a_{+}\left(v\left(h_{+}, t^{n+1}\right)-v\left(h_{+}, t^{n}\right)-v\left(0, t^{n+1}\right)+v\left(0, t^{n}\right)\right) \frac{h_{+}}{8} \\
& +b_{+}\left(v\left(h_{+}, t^{n+1}\right)-v\left(h_{+}, t^{n}\right)-v\left(0, t^{n+1}\right)+v\left(0, t^{n}\right)\right) \frac{k}{4} .
\end{aligned}
$$

Using algebraic manipulation, we obtain the following approximation of the modified model at the interface:

$$
\left(1+\lambda_{+}\right) v_{0+}^{n+1}=\left(1-\lambda_{+}\right) v_{0+}^{n}+\left(1+\lambda_{+}\right) v_{1}^{n}-\left(1-\lambda_{+}\right) v_{1}^{n+1}
$$

which is a second-order approximation to (2.18) at the interface. To update $v_{0-}^{n+1}$, we use the continuity condition

$$
v_{0+}^{n+1}=v_{0-}^{n+1}
$$


For the approximations at the interior points, we can use some well-known schemes to complete the computation. For example, we use Leap Frog (LF) and Lax-Wendroff (LW) schemes. For LF, we have

Scheme II.

$$
\begin{gathered}
a_{\operatorname{sign}(j)} \frac{v_{j}^{n+1}-v_{j}^{n-1}}{2 k}=b_{\operatorname{sign}(j)} \frac{v_{j+1}^{n}-v_{j-1}^{n}}{2 h_{\operatorname{sign}(j)}}, \quad j \neq 0, \\
v_{0-}^{n+1}=v_{0+}^{n+1}, \\
\left(1+\lambda_{+}\right) v_{0+}^{n+1}=\left(1-\lambda_{+}\right) v_{0+}^{n}+\left(1+\lambda_{+}\right) v_{1}^{n}-\left(1-\lambda_{+}\right) v_{1}^{n+1} .
\end{gathered}
$$

Using LW, we then have

\section{Scheme III.}

$$
\begin{gathered}
v_{j}^{n+1}=\frac{\lambda_{\operatorname{sign}(j)}^{2}-\lambda_{\operatorname{sign}(j)}}{2} v_{j-1}^{n}+\left(1-\lambda_{\operatorname{sign}(j)}^{2}\right) v_{j}^{n} \\
+\frac{\lambda_{\operatorname{sign}(j)}^{2}+\lambda_{\operatorname{sign}(j)}}{2} v_{j+1}^{n}, \quad j \neq 0, \\
v_{0-}^{n+1}=v_{0+}^{n+1}, \\
\left(1+\lambda_{+}\right) v_{0+}^{n+1}=\left(1-\lambda_{+}\right) v_{0+}^{n}+\left(1+\lambda_{+}\right) v_{1}^{n}-\left(1-\lambda_{+}\right) v_{1}^{n+1} .
\end{gathered}
$$

Like the first scheme, these two schemes are also semi-implicit. The stability for these schemes will be proved in the next section.

Remark 2. Since the condition that the physical and numerical interfaces are at the same point, $x=0$, is not a crucial assumption in the derivation of the three schemes above, they can be easily extended to treat the cases with multiple interfaces, in which the physical and numerical interfaces may or may not be at the same places.

Remark 3. The ideas used in deriving the above schemes can be extended to the cases in which the coefficients are functions of $x$. For example, let us consider

$$
\begin{gathered}
a(x) u_{t}(x, t)=(b(x) u(x, t))_{x}, \\
u(x, 0)=f(x),
\end{gathered}
$$

with the interface condition

$$
b(0-) u(0-, t)=b(0+) u(0+, t) .
$$

We derive the following scheme for this variable-coefficient problem. It is similar to Scheme II.

$$
a_{j} \frac{u_{j}^{n+1}-u_{j}^{n-1}}{2 k}=\frac{b_{j+1} u_{j+1}^{n}-b_{j-1} u_{j-1}^{n}}{2 h_{\operatorname{sign}(j)}}, \quad j \neq 0,
$$




$$
\begin{gathered}
b(0-) u_{0-}^{n+1}=b(0+) u_{0+}^{n+1}, \\
\left(u_{0+}^{n+1}-u_{0+}^{n}\right) \frac{a_{1 / 2}+a_{0+}}{2} \frac{h_{+}}{2}+\left(u_{1}^{n+1}-u_{1}^{n}-u_{0+}^{n+1}+u_{0+}^{n}\right) \frac{a_{1 / 2} h_{+}}{8} \\
=\left(b_{1} u_{1}^{n}-b_{0+} u_{0+}^{n}\right) \frac{k}{2}-\left(a_{1}\left(u_{1}^{n+1}-u_{1}^{n}\right)-a_{0+}\left(u_{0+}^{n+1}-u_{0+}^{n}\right)\right) \frac{h_{+}}{8} \\
+\left(b_{1}\left(u_{1}^{n+1}-u_{1}^{n}\right)-b_{0+}\left(u_{0+}^{n+1}-u_{0+}^{n}\right)\right) \frac{k}{4} .
\end{gathered}
$$

Schemes similar to Scheme I and Scheme III for the first-order equations can be derived for variable coefficients by similar arguments.

For the applications to the second-order acoustic wave equation with piecewise constant coefficients, we consider the following simple case with one interface:

$$
\begin{gathered}
u_{t t}(x, t)=c_{\operatorname{sign}(x)}^{2} u_{x x}(x, t), \quad x \neq 0, t>0, \\
u(x, 0)=g(x), \\
u_{t}(x, t)=f(x)
\end{gathered}
$$

and the physical interface conditions

$$
\begin{aligned}
u(0-, t) & =u(0+, t), \\
c_{-}^{2} u_{x}(0-, t) & =c_{+}^{2} u_{x}(0+, t) .
\end{aligned}
$$

In order to apply the ideas for first-order equations to this second-order problem, we decompose it into a first-order system as follows. Let

$$
\begin{aligned}
& v(x, t)=u_{t}(x, t)+c_{\operatorname{sign}(x)} u_{x}(x, t), \\
& w(x, t)=u_{t}(x, t)-c_{\operatorname{sign}(x)} u_{x}(x, t) .
\end{aligned}
$$

Then $v(x, t)$ and $w(x, t)$ satisfy

$$
\begin{gathered}
v_{t}(x, t)-c_{\operatorname{sign}(x)} v_{x}(x, t)=0, \\
w_{t}(x, t)+c_{\operatorname{sign}(x)} w_{x}(x, t)=0, \\
v(x, 0)=f(x)+c_{\operatorname{sign}(x)} g^{\prime}(x), \\
w(x, 0)=f(x)-c_{\operatorname{sign}(x)} g^{\prime}(x),
\end{gathered}
$$

and interface conditions (2.39) and (2.40). Then, we derive the following scheme for equations $(2.43)-(2.46)$ that is similar to Scheme III. 
Scheme IV.

$$
\begin{aligned}
\left(\begin{array}{c}
v_{j}^{n+1} \\
w_{j}^{n+1}
\end{array}\right)= & \left(\begin{array}{cc}
\frac{\sigma_{\mathrm{sign}(j)}^{2}-\sigma_{\mathrm{sign}(j)}}{2} & 0 \\
0 & \frac{\sigma_{\mathrm{sign}(j)}^{2}+\sigma_{\mathrm{sign}(j)}}{2}
\end{array}\right)\left(\begin{array}{c}
v_{j-1}^{n} \\
w_{j-1}^{n}
\end{array}\right) \\
& +\left(\begin{array}{cc}
1-\sigma_{\operatorname{sign}(j)}^{2} & 0 \\
0 & 1-\sigma_{\mathrm{sign}(j)}^{2}
\end{array}\right)\left(\begin{array}{c}
v_{j}^{n} \\
w_{j}^{n}
\end{array}\right) \\
& +\left(\begin{array}{cc}
\frac{\sigma_{\mathrm{sign}(j)}^{2}+\sigma_{\mathrm{sign}(j)}}{2} & 0 \\
0 & \frac{\sigma_{\mathrm{sign}(j)}^{2}-\sigma_{\mathrm{sign}(j)}}{2}
\end{array}\right)\left(\begin{array}{c}
v_{j+1}^{n} \\
w_{j+1}^{n}
\end{array}\right),
\end{aligned}
$$

$$
\left(1+\sigma_{+}\right) v_{0+}^{n+1}=\left(1-\sigma_{+}\right) v_{0+}^{n}+\left(1+\sigma_{+}\right) v_{1}^{n}-\left(1-\sigma_{+}\right) v_{1}^{n+1}
$$

$$
\left(1+\sigma_{-}\right) w_{0-}^{n+1}=\left(1-\sigma_{-}\right) w_{0-}^{n}+\left(1-\sigma_{-}\right) w_{-1}^{n}-\left(1-\sigma_{-}\right) w_{-1}^{n+1}
$$

$$
w_{0+}^{n+1}=v_{0+}^{n+1}-\frac{2 c_{-}}{c_{-}+c_{+}}\left(v_{0+}^{n+1}-w_{0-}^{n+1}\right),
$$

$$
v_{0-}^{n+1}=w_{0-}^{n+1}+\frac{2 c_{+}}{c_{-}+c_{+}}\left(v_{0+}^{n+1}-w_{0-}^{n+1}\right),
$$

where

$$
\sigma_{ \pm}=\frac{c_{ \pm} k}{h_{ \pm}}
$$

This scheme is also semi-implicit, and its stability requirements will be obtained in the next section.

Remark 4. As in Remark 1, if we apply the integral identity idea to (2.36) and use the simple first-order difference approximations, then we obtain a scheme which gives the exact value of the solution to the wave equation $(2.36)-(2.38)$ under the conditions that $\sigma_{ \pm}=1$. In fact, as in Remark 1, we can define the scheme as follows:

$$
\begin{aligned}
u_{j}^{n+1}-2 u_{j}^{n}+u_{j}^{n-1} & =\sigma_{ \pm}\left(u_{j+1}^{n}-2 u_{j}^{n}+u_{j-1}^{n}\right) \\
u_{0-}^{n+1}= & u_{0+}^{n+1} \\
\frac{c_{+}^{2}}{h_{+}}\left(u_{1}^{n}-u_{0+}^{n}\right)-\frac{c_{-}^{2}}{h_{-}}\left(u_{0-}^{n}-u_{-1}^{n}\right)= & \frac{h_{-}}{2 k^{2}}\left(u_{0-}^{n+1}-2 u_{0-}^{n}+u_{0-}^{n-1}\right) \\
& +\frac{h_{+}}{2 k^{2}}\left(u_{0+}^{n+1}-2 u_{0+}^{n}+u_{0+}^{n-1}\right) .
\end{aligned}
$$


If $g(x)=0$ and $f(x)$ has compact support on the left-hand side of the interface, then the solution to $(2.36)-(2.40)$ is given by

$$
u(x, t)=\left\{\begin{aligned}
\frac{1}{2}\left(f\left(x+c_{-} t\right)+f\left(x-c_{-} t\right)\right) & \\
+\frac{c_{-}-c_{+}}{2\left(c_{-}+c_{+}\right)} f\left(-x-c_{-} t\right), & x<0, \\
\frac{c_{-}}{c_{-}+c_{+}} f\left(\frac{c_{-}}{c_{+}}\left(x-c_{+} t\right)\right), & x>0 .
\end{aligned}\right.
$$

Since $\sigma_{ \pm}=1$, the values of $u(x, t)$ at the grid points become

$$
u\left(j h_{\mathrm{sign}(j)}, n k\right)= \begin{cases}\frac{1}{2}\left(f\left((j+n) h_{-}\right)+f\left((j-n) h_{-}\right)\right) & \\ +\frac{c_{-}-c_{+}}{2\left(c_{-}+c_{+}\right)} f\left((-j-n) h_{-}\right), & j<0, \\ \frac{c_{-}}{c_{-}+c_{+}} f\left((j-n) h_{-}\right), & j>0 .\end{cases}
$$

By substituting (2.55) into (2.53), we can easily see that the values of the solution to (2.36)-(2.40) on the grids satisfy the above difference equations. In other words, we have shown that these difference equations give us the exact value of the analytic solution of (2.36)-(2.40) for any number of physical interfaces, if for fixed $k$ we can keep $\sigma=1$ at each grid point. If the integration done here and in Remark 1 is applied to the elastic equation of $\S 1$, the analytic solution for any number of interfaces is also obtained (if $\sigma=1$ and $k$ is fixed).

\section{Stability analysis}

In this section, we state and prove several stability results for the schemes derived in the last section. The results are based on stability theorems of Trefethen [10], which are extensions of the well-known 'GKS' stability theorems [7].

Throughout this section, we use $u_{j}^{n}$ only to indicate the solutions to Schemes I-III. In order to apply Trefethen's theory, we begin with a discussion of the general solutions to Schemes I-III of the form $u_{j}^{n}=z^{n} \phi_{j}$. By substituting this solution into Schemes I-III, we see that it can be represented as

$$
u_{j}^{n}=\rho_{1 \operatorname{sign}(j)} z^{n} \chi_{1 \operatorname{sign}(j)}^{j}+\rho_{2 \operatorname{sign}(j)} z^{n} \chi_{2 \operatorname{sign}(j)}^{j},
$$

where $\chi_{1 \pm}$ and $\chi_{2 \pm}$ are either the roots of

$$
\chi^{2}-\frac{z^{2}-1}{\lambda_{ \pm} z} \chi-1=0
$$

or the roots of

$$
z \chi=\chi+\frac{\lambda_{ \pm}}{2}\left(\chi^{2}-1\right)+\frac{\lambda_{ \pm}^{2}}{2}(\chi-1)^{2}
$$


We classify the wave components $z^{n} \chi_{1 \operatorname{sign}(j)}^{j}$ and $z^{n} \chi_{2 \operatorname{sign}(j)}^{j}$ in (3.1) into leftgoing and right-going waves, according to their group velocities, which are defined [11] as

$$
C_{ \pm}^{i}=-\left.\frac{1}{\lambda_{ \pm}} \frac{\chi_{i \pm}}{z} \frac{d z_{0}}{d \chi}\right|_{z_{0}=z, \chi=\chi_{i \pm}}, \quad i=1,2,
$$

where $z_{0}$ and $\chi$ satisfy (3.2) or (3.3), with corresponding $\lambda_{ \pm}$and $i$. The definitions from [11] are

Definition 1. For $i=1,2$, if $\left|\chi_{i+}\right|=|z|=1$ and its corresponding $C_{+}^{i} \leq 0$ $\left(C_{+}^{i} \geq 0\right)$, then $z^{n} \chi_{i+}^{j}$ is called left-going (right-going). Also, if $|z| \geq 1,\left|\chi_{i+}\right|>$ $1\left(|z| \geq 1,\left|\chi_{i+}\right|<1\right)$, then $z^{n} \chi_{i+}^{j}$ is called left-going (right-going), independent of $C_{+}^{i}$. For $z^{n} \chi_{i-}^{j}$ we have the same definitions.

In order to classify the wave components, we need the following lemmas, which are due to Gustafsson, Kreiss, and Sundström [7].

Lemma 1. The roots of (3.2) have the following properties: if $|z|>1$, then $\left|\chi_{1+}\right|<1,\left|\chi_{2+}\right|>1$; if $z=e^{i \theta}$, then

$$
\begin{aligned}
& \left|\chi_{1+}\right|<1, \quad\left|\chi_{2+}\right|>1 \quad \text { for }|\sin \theta|>\lambda_{+}, \\
& \left|\chi_{1+}\right|=1, \quad\left|\chi_{2+}\right|=1 \quad \text { for }|\sin \theta| \leq \lambda_{+} \text {, } \\
& \chi_{1+}=-1, \quad \chi_{2+}=1 \quad \text { for } \theta=0 \text {, } \\
& \chi_{1+}=1, \quad \chi_{2+}=-1 \text { for } \theta=\pi \text {, } \\
& \chi_{1+}=\chi_{2+}= \pm i \quad \text { for } \sin \theta= \pm \lambda_{+} \text {. }
\end{aligned}
$$

The same conclusions hold for $\chi_{1_{-}}, \chi_{2_{-}}$, and the corresponding $\lambda_{-}$.

Lemma 2. There exists a $\delta>0$ such that the two roots of (3.3), $\chi_{1+}$ and $\chi_{2+}$ for $\lambda_{+}$, satisfy

I. if $\lambda_{+}>0$, then

$$
\begin{array}{ll}
\left|\chi_{1+}\right| \leq 1-\delta & \text { for }|z| \geq 1, \\
\left|\chi_{2+}\right|>1 & \text { for }|z| \geq 1, \quad z \neq 1, \\
\chi_{2+}=1 & \text { for } z=1,
\end{array}
$$

II. if $\lambda_{+}<0$, then

$$
\begin{aligned}
\left|\chi_{1+}\right|<1 & \text { for }|z| \geq 1, \quad z \neq 1 \\
\chi_{1+}=1 & \text { for } z=1 \\
\left|\chi_{2+}\right| \geq 1+\delta & \text { for }|z| \geq 1 .
\end{aligned}
$$

The same is true for $\chi_{1-}$ and $\chi_{2-}$ with the corresponding $\lambda_{-}$. 
Using the above definition and lemmas, we prove

Lemma 3. For Schemes I-III, $z^{n} \chi_{1+}^{j}, z^{n} \chi_{1-}^{j}$ are right-going and $z^{n} \chi_{2+}^{j}, z^{n} \chi_{2-}^{j}$ are left-going.

Proof. If $z^{n} \chi_{1 \operatorname{sign}(j)}^{j}$ and $z^{n} \chi_{2 \operatorname{sign}(j)}^{j}$ are the wave components for the general solution $z^{n} \phi_{j}$ to Schemes I and II, then $\chi_{1 \pm}, \chi_{2 \pm}$ are solutions of equation (3.2). If $|z|>1$, then by Lemma 1 we know that $z^{n} \chi_{1 \pm}^{j}$ are right-going and $z^{n} \chi_{2 \pm}^{j}$ are left-going. If $z=e^{i \theta}$ and $|\sin (\theta)|>\lambda_{ \pm}$, then we have the same conclusion by Lemma 1 . If $z=e^{i \theta}$ and $|\sin (\theta)| \leq \lambda_{ \pm}$, then by Lemma 1 , $\left|\chi_{1 \pm}\right|=\left|\chi_{2 \pm}\right|=1$. Since $\chi_{1 \pm} \chi_{2 \pm}=-1$, we have

$$
\operatorname{Re} \chi_{1 \pm} \operatorname{Re} z \leq 0 \leq \operatorname{Re} \chi_{2 \pm} \operatorname{Re} z
$$

then by (2.4) of [11] we know that the group velocities of these wave components satisfy

$$
\begin{aligned}
& C_{ \pm}^{1}=-\frac{\chi_{1 \pm}+1 / \chi_{1 \pm}}{z+1 / z}=-\frac{\operatorname{Re} \chi_{1 \pm}}{\operatorname{Re} z} \geq 0, \\
& C_{ \pm}^{2}=-\frac{\chi_{2 \pm}+1 / \chi_{2 \pm}}{z+1 / z}=-\frac{\operatorname{Re} \chi_{2 \pm}}{\operatorname{Re} z} \leq 0 .
\end{aligned}
$$

By the definition, $z^{n} \chi_{1 \pm}^{j}$ are right-going and $z^{n} \chi_{2 \pm}^{j}$ are left-going. The results for Schemes I and II are then verified. If $z^{n} \chi_{1 \pm}^{j}$ and $z^{n} \chi_{2 \pm}^{j}$ are the wave components of the general solution $z^{n} \phi_{j}$ to Scheme III, $\chi_{1 \pm}, \chi_{2 \pm}$ are solutions of equation (3.3). In this case, if $|z| \geq 1$ and $z \neq 1$, then Lemma 2 guarantees the results of this lemma. If $z=1$, then by Lemma $2, z^{n} \chi_{1 \pm}^{j}$ are right-going. For $z^{n} \chi_{2 \pm}^{j}$, we consider its group velocity. Let $z_{0}$ be the function of $\chi$ defined by equation (3.3); then one can easily see that $d z_{0} /\left.d \chi\right|_{z_{0}=1, \chi=1}=1$. Thus, by (3.4) and Lemma 2, we know that $C_{ \pm}^{2}=-1 / \lambda_{ \pm} \leq 0, z^{n} \chi_{2 \pm}^{j}$ are left-going. The results for Scheme III are then verified.

Definition 2. The general solutions $u_{j}^{n}$ to the schemes are called purely outgoing from the interface if there are no wave components moving toward the interface.

We now discuss GKS-stability for the schemes. The results below are mainly based on Trefethen's group velocity version of the GKS-stability theorem. For completeness, we state Trefethen's stability result [10] as follows.

Theorem 1. A difference model for an initial value problem is GKS-unstable if and only if it admits a solution $z^{n} \phi_{j}$ which is purely outgoing from the interface.

The first stability theorem is for Scheme I with constant coefficients and a uniform grid size.

Theorem 2. If $a_{+}=a_{-}, b_{+}=b_{-}$, and $h_{+}=h_{-}=h$, then Scheme I is GKSunstable for any $\lambda_{+}=\lambda_{-}=\lambda>0$. Moreover, if $\lambda_{+}=\lambda_{-}=\lambda>\sqrt{15} / 4$, then Scheme I is even $l_{2}$-unstable. 
Proof. In order to find a purely outgoing solution to Scheme I, we substitute

$$
u_{j}^{n}= \begin{cases}\rho_{1+} z^{n} \chi_{1+}^{j}, & j \geq 0, \\ \rho_{2-} z^{n} \chi_{2-}^{j}, & j \leq 0,\end{cases}
$$

into the interface conditions (2.9) and (2.10) of Scheme I. After some simplifications, we end up with

$$
\rho_{1+}-\rho_{2-}=0 \text {, }
$$

and

$$
\left[\frac{\left(z^{2}-1\right)}{16 \lambda}\left(\chi_{1+}+3\right)-\frac{1}{2} z \chi_{1+}\right] \rho_{1+}+\left[\frac{\left(z^{2}-1\right)}{16 \lambda}\left(\frac{1}{\chi_{2-}}+3\right)+\frac{z}{2 \chi_{2-}}\right] \rho_{2-}=0 .
$$

Note that the conditions $a_{-}=a_{+}, b_{-}=b_{+}$, and $h_{-}=h_{+}$are used in the above equation. To obtain nonzero $\rho_{1+}$ and $\rho_{2-}$, i.e., nonzero $u_{j}^{n}$ defined by (3.5), we must have

$$
\mid \frac{\left(z^{2}-1\right)}{16 \lambda}\left(\begin{array} { c } 
{ 1 } \\
{ \chi _ { 1 + } + 3 ) - \frac { z \chi _ { 1 + } } { 2 } }
\end{array} \frac { ( z ^ { 2 } - 1 ) } { 1 6 \lambda } \left(\begin{array}{c}
-1 \\
\left.\frac{1}{\chi_{2-}}+3\right)+\frac{z}{2 \chi_{2-}}
\end{array} \mid=0 .\right.\right.
$$

This yields

$$
\frac{\left(z^{2}-1\right)}{16 \lambda}\left(\chi_{1+}+\frac{1}{\chi_{2-}}+6\right)-\frac{z}{2}\left(\chi_{1+}-\frac{1}{\chi_{2-}}\right)=0 .
$$

Since $\lambda_{-}=\lambda_{+}=\lambda$ in (3.2), we have $\chi_{1+}=-1 / \chi_{2-}$. Then,

$$
z^{2}-1=\frac{8}{3} \lambda z \chi_{1+} \text {. }
$$

Solving for $\chi_{1+}$ and $z$, using this equation and equation (3.2), gives

$$
z= \pm \frac{8 \lambda}{2 \sqrt{15}} i \pm \frac{\sqrt{60-64 \lambda^{2}}}{2 \sqrt{15}}, \quad \chi_{1+}= \pm \frac{\sqrt{3}}{\sqrt{5}} i, \quad \chi_{2-}=\mp \frac{\sqrt{5}}{\sqrt{3}} i .
$$

For these $z, \chi_{1+}$, and $\chi_{2-}$, we can choose $\rho_{1+}=\rho_{2-}=1$ so that $u_{j}^{n}$ defined by (3.5) is a purely outgoing solution to Scheme I, since we can easily see that $|z|=1$ for the $z$ chosen as above. Hence, Scheme I is GKS-unstable, according to Theorem 1 .

Moreover, if $\lambda_{-}=\lambda_{+}=\lambda>\sqrt{15} / 4$, we can easily see that

$$
z=\frac{8 \lambda i}{2 \sqrt{15}}+\frac{\sqrt{60-64 \lambda^{2}}}{2 \sqrt{15}}
$$

satisfies $|z|>1$; thus, the corresponding $u_{j}^{n}$ in (3.5) contains strictly outgoing eigensolutions of Scheme I. By the Godunov-Ryabenkii theorem [12], Scheme I is $l_{2}$-unstable if $\lambda_{+}=\lambda_{-}=\lambda>\sqrt{15} / 4$.

Remark 1 . Since GKS-instability usually does not imply $l_{2}$-instability [11], we conjecture that Scheme I is $l_{2}$-stable if the conditions in Theorem 2 are satisfied and $\lambda \leq \sqrt{15} / 4$. Our numerical experiments support this conjecture. For the 
general case with piecewise constant coefficients and nonuniform space steps, our numerical experiments suggest that Scheme I is stable when $\max \left(\lambda_{-}, \lambda_{+}\right) \leq$ $\sqrt{15} / 4$.

Theorem 2 characterizes the stability of Scheme I under rather strict conditions, but the results are very interesting. Under the conditions of Theorem 2 , we can use LF to approximate the PDE, and the stability for the LF scheme is $\lambda \leq 1$; however, the interface scheme in Scheme I requires that $\lambda \leq \sqrt{15} / 4$ for Scheme I to be stable under the conditions of Theorem 2 . This shows that interfaces (physical and/or numerical) may require more restrictive stability conditions than normal interior points. In particular, it is important to note that a CFL condition of unity yields an unstable interface condition!

Theorem 3. Scheme II is GKS-stable for any $\lambda_{ \pm}$satisfying $0<\lambda_{ \pm} \leq 1$.

Proof. We prove this theorem by showing that Scheme II does not allow any purely outgoing solutions. Suppose it does; then, by substituting $u_{j}^{n}$, defined in (3.5), into the interface equations of Scheme II, we have

$$
\rho_{1+}-\rho_{2-}=0
$$

and

$$
\left(1+\lambda_{+}\right) z \rho_{1+}-\left[\left(1-\lambda_{+}\right)+\left(1+\lambda_{+}\right) \chi_{1+}-\left(1-\lambda_{+}\right) z \chi_{1+}\right] \rho_{1+}=0 .
$$

If $\left(\rho_{1+}, \rho_{2-}\right)$ is not zero, then we must have

$$
\left(1+\lambda_{+}\right) z=\left(1-\lambda_{+}\right)+\left(1+\lambda_{+}\right) \chi_{1+}-\left(1-\chi_{+}\right) z \chi_{1+},
$$

or, equivalently,

$$
\chi_{1+}=\frac{\left(1-\lambda_{+}\right)-\left(1+\lambda_{+}\right) z}{\left(1-\lambda_{+}\right) z-\left(1+\lambda_{+}\right)} .
$$

Solving this together with

$$
\chi_{1+}^{2}-\frac{z^{2}-1}{\lambda_{+} z} \chi_{1+}-1=0
$$

for $z$ and $\chi_{1+}$, we have $z= \pm 1$ and the corresponding $\chi_{1+}= \pm 1$. But, according to Lemma 1 , we must have $\chi_{1+}=-1$ when $z=1$ and $\chi_{1+}=1$ when $z=-1$. This contradiction tells us that Scheme II has no purely outgoing solution; hence, it is GKS-stable by Theorem 1 .

To prove the stability of Scheme III, we need the following lemma.

Lemma 4. For $0<\lambda \leq 1$, the function

$$
w(z)=\frac{(1-\lambda)-(1+\lambda) z}{(1-\lambda) z-(1+\lambda)}
$$

maps the unit circle of the complex plane to itself and maps the inside of the unit circle to the inside, the outside to the outside.

Proof. These results follow from the fact that $w(z)$ is a Möbius mapping. 
Theorem 4. Scheme III is GKS-stable for any $\lambda_{ \pm}$satisfying $0<\lambda_{ \pm} \leq 1$.

Proof. If $u_{j}^{n}$ given by (3.5) is a purely outgoing solution to Scheme III, then by substituting it into the interface equations of Scheme III we have

$$
\rho_{1+}-\rho_{2-}=0 \text {, }
$$

and

$$
\left(1+\lambda_{+}\right) z \rho_{1+}-\left[\left(1-\lambda_{+}\right)+\left(1+\lambda_{+}\right) \chi_{1+}-\left(1-\lambda_{+}\right) z \chi_{1+}\right] \rho_{1+}=0 .
$$

The existence of nonzero $\left(\rho_{1+}, \rho_{2-}\right)$ requires that

$$
\left(1+\lambda_{+}\right) z=\left(1-\lambda_{+}\right)+\left(1+\lambda_{+}\right) \chi_{1+}-\left(1-\lambda_{+}\right) z \chi_{1+},
$$

or, equivalently,

$$
\chi_{1+}=\frac{\left(1-\lambda_{+}\right)-\left(1+\lambda_{+}\right) z}{\left(1-\lambda_{+}\right) z-\left(1+\lambda_{+}\right)} .
$$

Hence, we must have $\left|\chi_{1+}\right| \geq 1$ when $|z| \geq 1$, according to Lemma 4. But, by Lemma 2, we know that $\left|\chi_{1+}\right|<1-\delta$ when $|z| \geq 1$ for some $\delta>0$. This contradiction means that Scheme III admits no purely outgoing solution; hence, it is GKS-stable by Theorem 1.

If we apply the techniques used to derive Schemes I-III to hyperbolic systems, we obtain similar systems of finite difference equations, and the proofs for the stability of the systems of finite difference equations are essentially similar. To show how this can be done, we prove the stability of Scheme IV of the last section. We start with the general solutions to $(2.47)-(2.51)$ of the form $\vec{u}_{j}^{n}=z^{n} \vec{\phi}_{j}$,where

$$
\vec{u}_{j}^{n}=\left(\begin{array}{c}
v_{j}^{n} \\
w_{j}^{n}
\end{array}\right),
$$

and $\vec{\phi}_{j}$ is a vector in $\mathbb{R}^{2}$. By substituting $\vec{u}_{j}^{n}$ into (2.47), we have

$$
\vec{u}_{j}^{n}=z^{n} \sum_{i=1}^{4} \rho_{i \operatorname{sign}(j)} \chi_{i \operatorname{sign}(j)}^{j} \vec{v}_{i},
$$

where the vectors $\vec{v}_{i}$ are

$$
\left(\begin{array}{l}
1 \\
0
\end{array}\right),\left(\begin{array}{l}
1 \\
0
\end{array}\right),\left(\begin{array}{l}
0 \\
1
\end{array}\right),\left(\begin{array}{l}
0 \\
1
\end{array}\right)
$$

$\chi_{1 \pm}$ and $\chi_{2 \pm}$ are solutions to

$$
z \chi=\chi+\frac{\sigma_{ \pm}}{2}\left(\chi^{2}-1\right)+\frac{\sigma_{ \pm}^{2}}{2}(\chi-1)^{2}
$$

and $\chi_{3 \pm}$ and $\chi_{4 \pm}$ are solutions to

$$
z \chi=\chi-\frac{\sigma_{ \pm}}{2}\left(\chi^{2}-1\right)+\frac{\sigma_{ \pm}^{2}}{2}(\chi-1)^{2} .
$$

The following lemma classifies the components of $\vec{u}_{j}^{n}$. 
Lemma 5. $z^{n} \chi_{1 \pm}^{j} \vec{v}_{1}$ and $z^{n} \chi_{3 \pm}^{j} \vec{v}_{3}$ are right-going, and $z^{n} \chi_{2 \pm}^{j} \vec{v}_{2}$ and $z^{n} \chi_{4 \pm}^{j} \vec{v}_{4}$ are left-going.

Proof. We choose $\chi_{1 \pm}$ and $\chi_{3 \pm}$ to $k 2$ the first roots of (3.7) and (3.8), and $\chi_{2 \pm}$ and $\chi_{4 \pm}$ to be the second roots of (3.7) and (3.8), respectively. By Lemma 2 and Definition 1, we can easily see that $z^{n} \chi_{1 \pm}^{j} \vec{v}_{1}$ are right-going and $z^{n} \chi_{4 \pm}^{j} \vec{v}_{4}$ are left-going. Also, if $z \neq 1$, then $z^{n} \chi_{2 \pm}^{j} \vec{v}_{2}$ are left-going and $z^{n} \chi_{3 \pm}^{j} \vec{v}_{3}$ are right-going. If $z=1$, then $\chi_{2 \pm}=1$ and $\chi_{3 \pm}=1$, and we can easily show that

$$
\left.\frac{d z_{0}}{d \chi}\right|_{z=1, \chi=1}=\sigma_{ \pm}>0
$$

when $z_{0}$ is a function of $\chi$ defined by (3.7). Similarly,

$$
\left.\frac{d z_{0}}{d \chi}\right|_{z=1, \chi=1}=-\sigma_{ \pm}<0
$$

when $z_{0}$ is a function of $\chi$ defined by (3.8). By (3.4), when $z=1$, the group velocity of $z^{n} \chi_{2 \pm}^{j} \vec{v}_{2}$ is -1 , and the group velocity of $z^{n} \chi_{3 \pm}^{j} \vec{v}_{3}$ is 1 . Hence, $z^{n} \chi_{2 \pm}^{j} \vec{v}_{2}$ and $z^{n} \chi_{3 \pm}^{j} \vec{v}_{3}$ are still left-going and right-going, respectively, when $z=1$.

Theorem 5. The scheme defined by (2.47)-(2.51) for the second-order hyperbolic equations is stable for $0<\sigma_{ \pm} \leq 1$.

Proof. According to Lemma 5, the purely outgoing solution to the scheme must have the following form:

$$
u_{j}^{n}= \begin{cases}\rho_{2-} z^{n} \chi_{2-}^{j} \vec{v}_{2}+\rho_{4-} z^{n} \chi_{4-}^{j} \vec{v}_{4}, & j \leq 0, \\ \rho_{1+} z^{n} \chi_{1+}^{j} \vec{v}_{1}+\rho_{3+} z^{n} \chi_{3+}^{j} \vec{v}_{3}, & j \geq 0 .\end{cases}
$$

Substituting $u_{j}^{n}$ into the interface equations of the scheme yields

$$
\begin{gathered}
\left(1-\frac{2 c_{-}}{c_{-}+c_{+}}\right) \rho_{1+}-\rho_{3+}+\frac{2 c_{-}}{c_{-}+c_{+}} \rho_{4-}=0, \\
\frac{2 c_{+}}{c_{-}+c_{+}} \rho_{1+}-\rho_{2-}+\left(1-\frac{2 c_{+}}{c_{-}+c_{+}}\right) \rho_{4-}=0, \\
\rho_{1+}\left[\left(1+\sigma_{+}\right) z-\left[\left(1-\sigma_{+}\right)+\left(1+\sigma_{+}\right) \chi_{1+}-\left(1-\sigma_{+}\right) z \chi_{1+}\right]\right]=0,
\end{gathered}
$$

and

$$
\rho_{4-}\left[\left(1+\sigma_{-}\right) z-\left[\left(1-\sigma_{-}\right)+\left(1+\sigma_{-}\right) \frac{1}{\chi_{4-}}-\left(1-\sigma_{-}\right) z \frac{1}{\chi_{4-}}\right]\right]=0 .
$$

Since $\left(\rho_{1+}, \rho_{2-}, \rho_{3+}, \rho_{4-}\right)$ is nonzero, we must have

$$
\left(1+\sigma_{+}\right) z-\left[\left(1-\sigma_{+}\right)+\left(1+\sigma_{+}\right) \chi_{1+}-\left(1-\sigma_{+}\right) z \chi_{1+}\right]=0
$$

and

$$
\left(1+\sigma_{-}\right) z-\left[\left(1-\sigma_{-}\right)+\left(1+\sigma_{-}\right) \frac{1}{\chi_{4-}}-\left(1-\sigma_{-}\right) z \frac{1}{\chi_{4-}}\right]=0 \text {. }
$$


This is equivalent to

$$
\chi_{1+}=\frac{\left(1-\sigma_{+}\right)-\left(1+\sigma_{+}\right) z}{\left(1-\sigma_{+}\right) z-\left(1+\sigma_{+}\right)}, \quad \frac{1}{\chi_{4-}}=\frac{\left(1-\sigma_{-}\right)-\left(1+\sigma_{-}\right) z}{\left(1-\sigma_{-}\right) z-\left(1+\sigma_{-}\right)} .
$$

Since the variable $z$ in our discussion satisfies $|z| \geq 1$, by Lemma 4 we should have $\left|\chi_{1+}\right| \geq 1$ and $\left|\chi_{4-}\right| \leq 1$. But, according to Lemma 2 , these are impossible. This contradiction means that this finite difference scheme does not admit any purely outgoing solution; hence, it is GKS-stable by Theorem 1.

\section{NUMERICAL EXAMPLES}

In this section, we apply the schemes derived in $\S 2$ to several examples and then make some comments.

Example 1. Theorem 2 shows that finite difference schemes with numerical interfaces usually have more restrictive stability conditions than those without numerical interfaces. In this example, we apply Scheme I to compute $u(x, t)$ in

$$
u_{t}(x, t)=u_{x}(x, t), \quad u(x, 0)=f(x)
$$

where

$$
f(x)=1000 \exp \left(\frac{1}{(x-0.15)(0.85-x)}\right) .
$$

We first use $h=0.01$ and $k=0.0096$ to compute $u(x, t)$ and plot the results in Figure 1 and Figure 2. Figure 1 is plotted before the wave peak hits the numerical interface at $x=0$. Figure 2 is plotted after the peak passes the interface. Since $\lambda_{+}=\lambda_{-}=\lambda<\sqrt{15} / 4$, the computation is stable. Now we choose $h=0.01$ and $k=0.01$. Since $\lambda_{+}=\lambda_{-}=1>\sqrt{15} / 4$, when the wave peak meets the interface the computation generates an instability that eventually blows up. This is plotted in Figure 3. To see that $\lambda_{+}=\lambda_{-}=\lambda \leq \sqrt{15} / 4$ is really necessary, we let $h_{ \pm}=0.01$ and $k=0.009683$; then $\lambda_{ \pm}=\lambda_{-}=\lambda=0.9683$, which is only slightly larger than $\sqrt{15} / 4$. After 1200 time steps, we can see the error build-up at $x=0$. This result is plotted in Figure 4. If we let $h_{ \pm}=0.01$ and $k=0.009682$, then $\lambda_{+}=\lambda_{-}=\lambda=0.9682$ is slightly smaller than $\sqrt{15} / 4$. The computational result at time step 1250 is plotted in Figure 5. The error does not accumulate at the interface $x=0$, due to the stability of the scheme.

Example 2. In this example, we apply Schemes I-III to the following problem:

$$
u_{t}(x, t)=b_{\operatorname{sign}(x)} u_{x}(x, t), \quad u(x, 0)=f(x)
$$

where

and

$$
b_{\operatorname{sign}(x)}= \begin{cases}1, & x<0 \\ 2, & x>0\end{cases}
$$

$$
f(x)=1000(x-0.15)^{4} \cdot(0.85-x)^{4}
$$




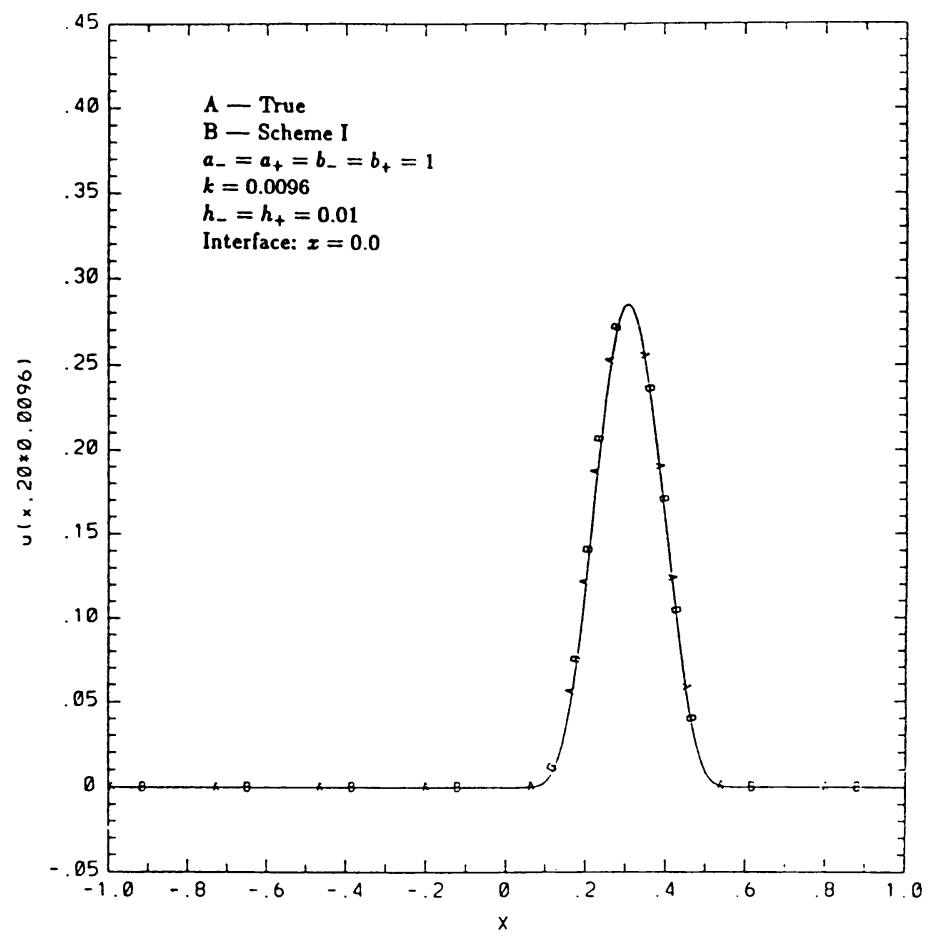

FIGURE 1

In addition to the physical interface, we introduce a numerical interface by letting $h_{+}=0.011, h_{-}=0.022$, and $k=0.01$. Figures 6-8 are the snapshots of the numerical results from Scheme I and the true solutions at the time levels before the wave peak reaches the interface, when the wave peak is at the interface, and after the wave peak has passed the interface. The accuracy is apparent, and the numerical results from Schemes II and III are as accurate.

Example 3. Even though Schemes I-III all seem to be able to handle the physical and numerical interface quite well, Scheme $I$ is less capable to treat the numerical interfaces than Schemes II and III. To see this, we apply Schemes I and II to the problem in Example 1. There is no physical interface in this example. We choose $h_{-}=0.04, h_{+}=0.0025, k=0.0024$. Figure 9 is the numerical result using Scheme I. The wave has passed the numerical interface $x=0$. In this picture, we see that Scheme I generates spurious waves at the interface, and these spurious waves have moved into the fine-grid region on the right-hand side. The numerical results from Scheme II are plotted in Figure 10. Scheme II nearly eliminates the spurious waves at the interface; the computational result in the fine-grid region is not contaminated. The schemes based on characteristics are seen to be more accurate in handling numerical interfaces. 
GRID REFINEMENT SCHEMES FOR HYPERBOLIC EQUATIONS

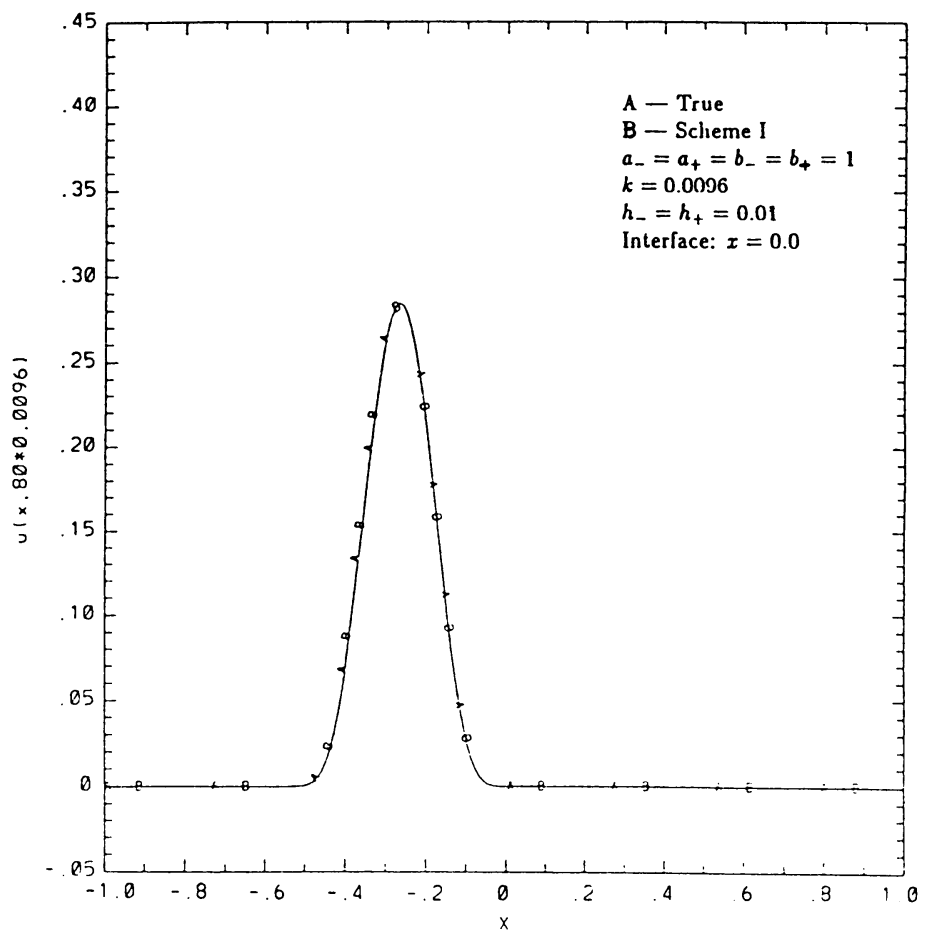

FIGURE 2

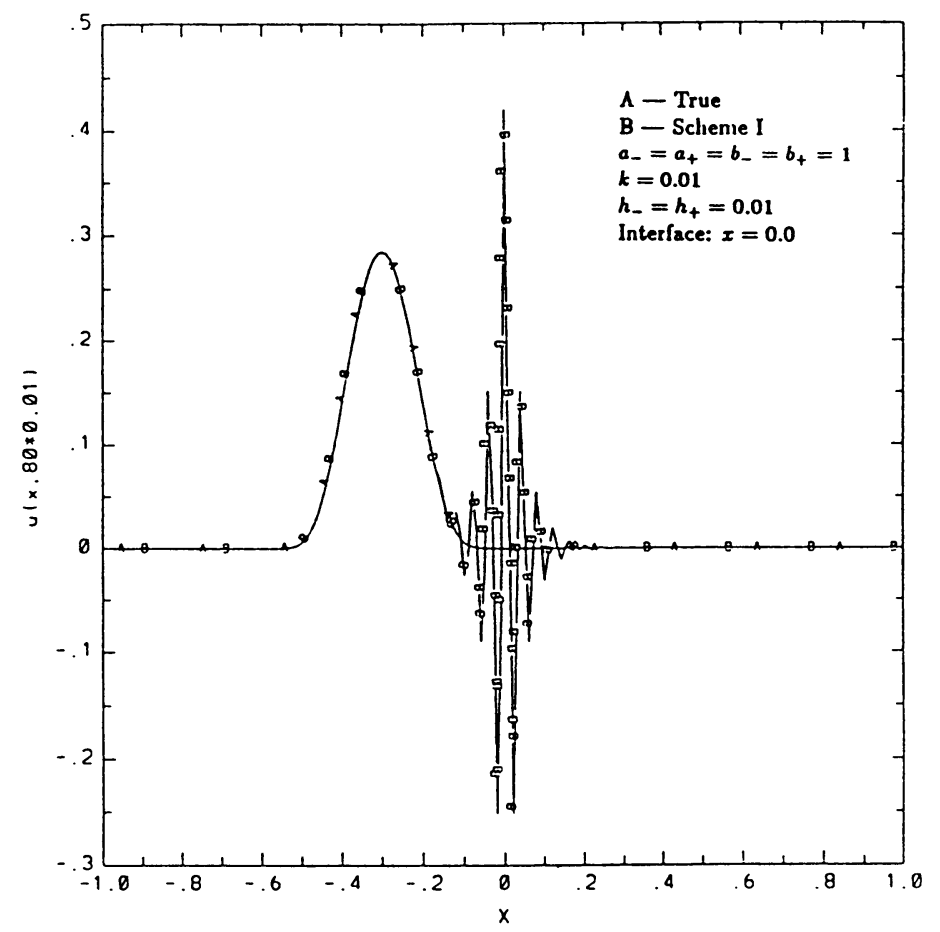

FIGURE 3 


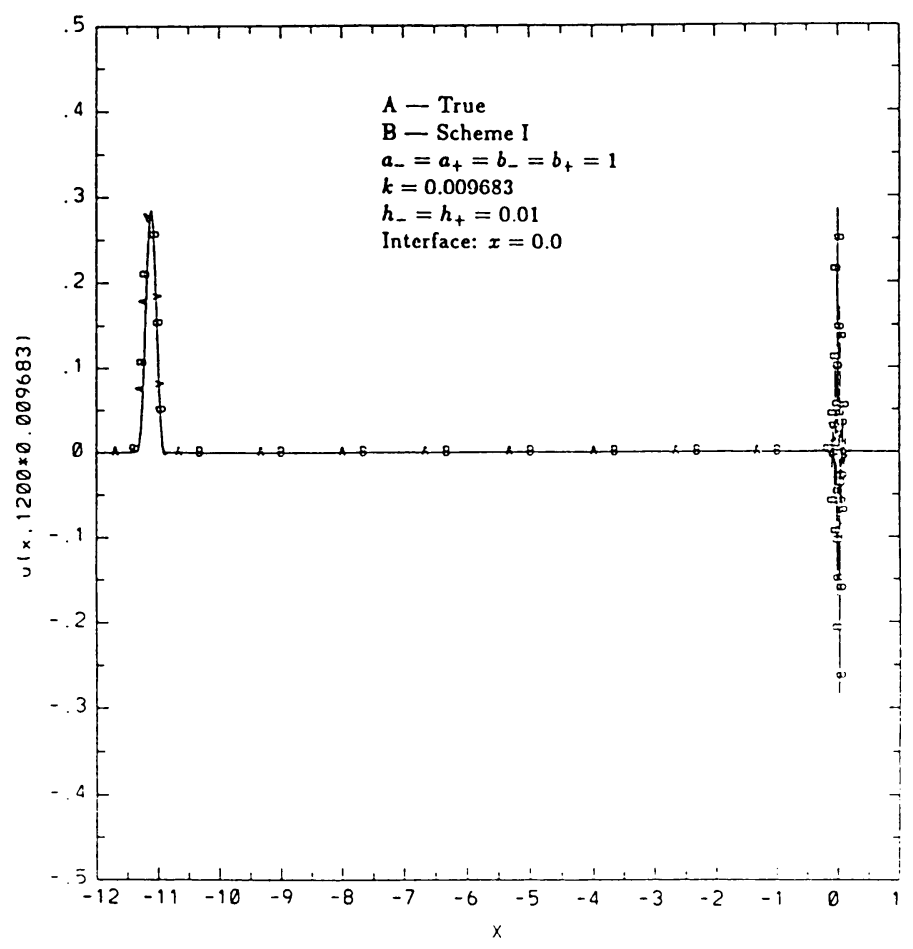

FIGURE 4

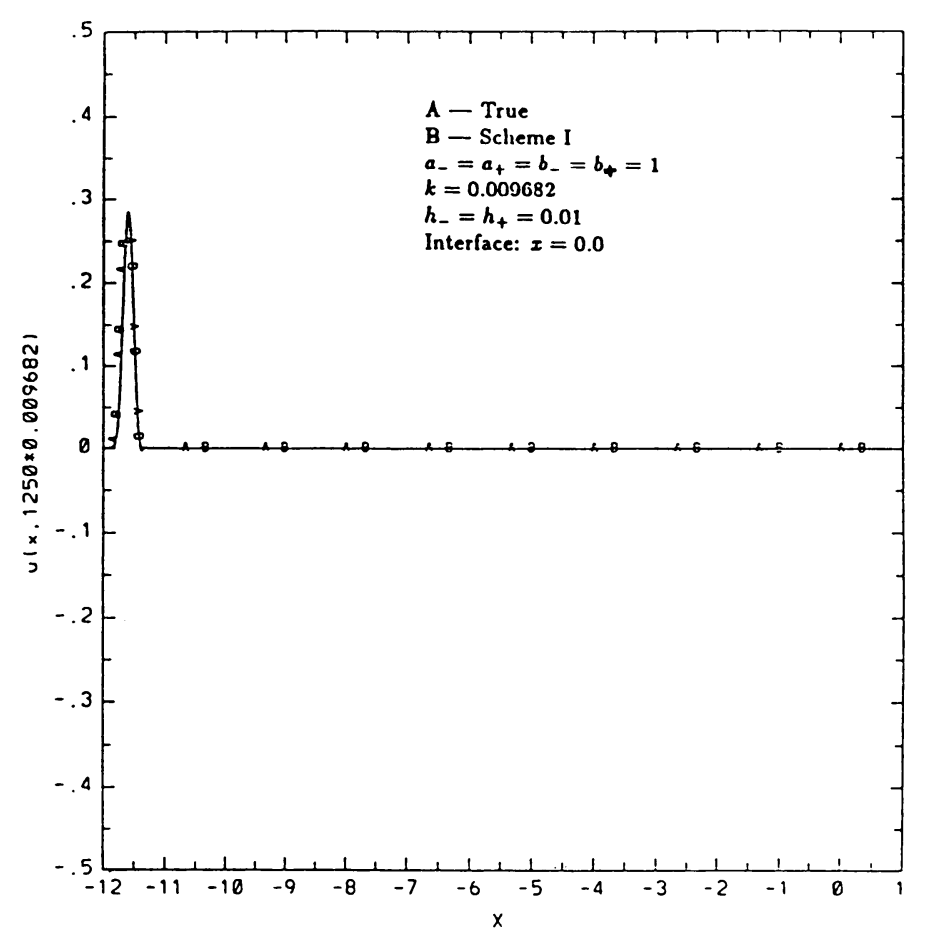

FIGURE 5 


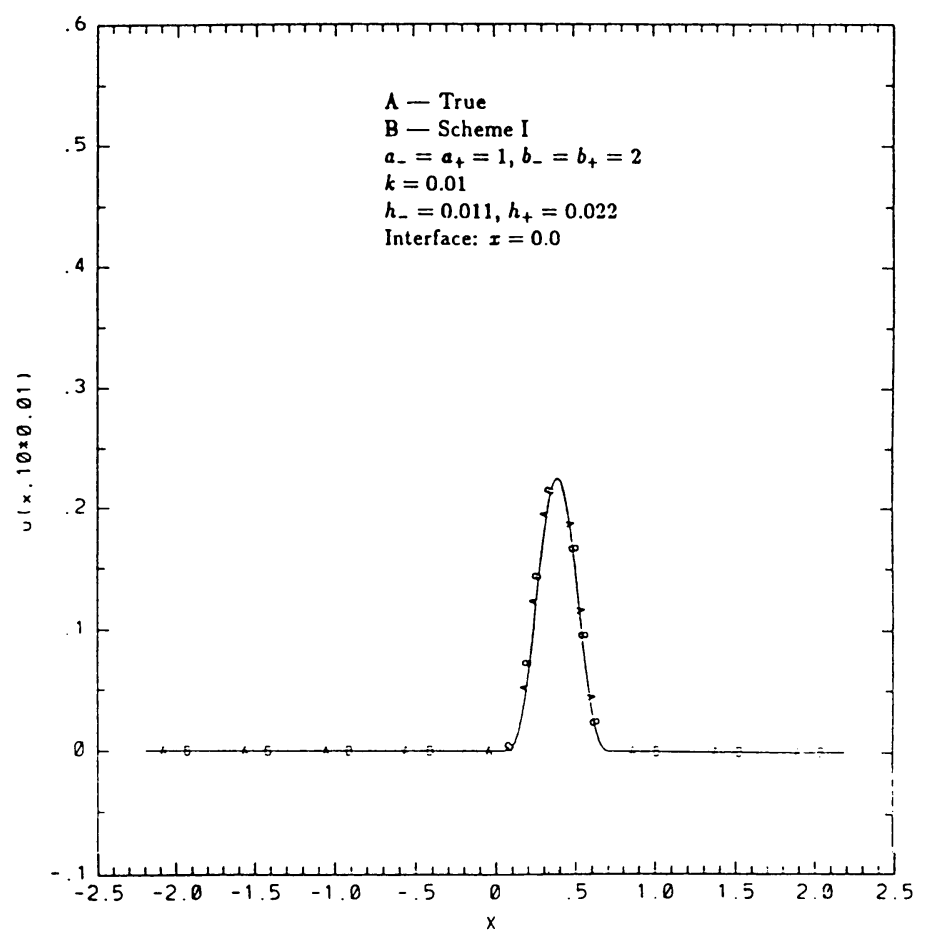

FIGURE 6

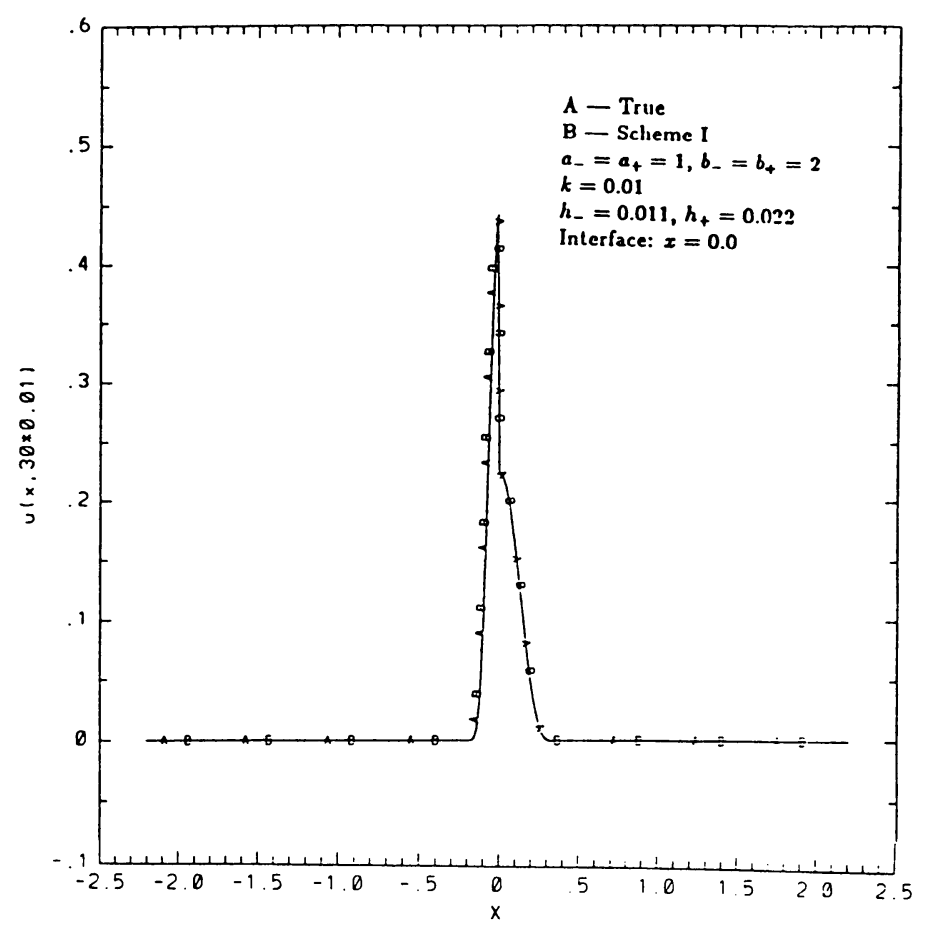

FIGURE 7 


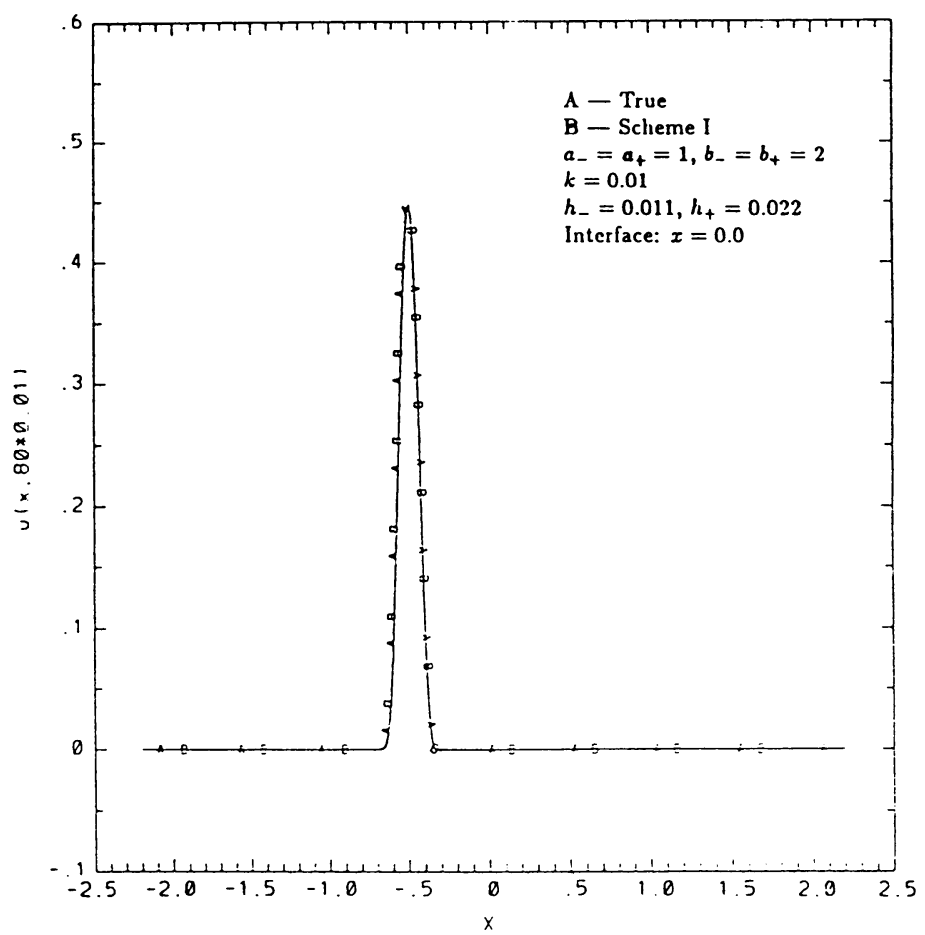

FIGURE 8

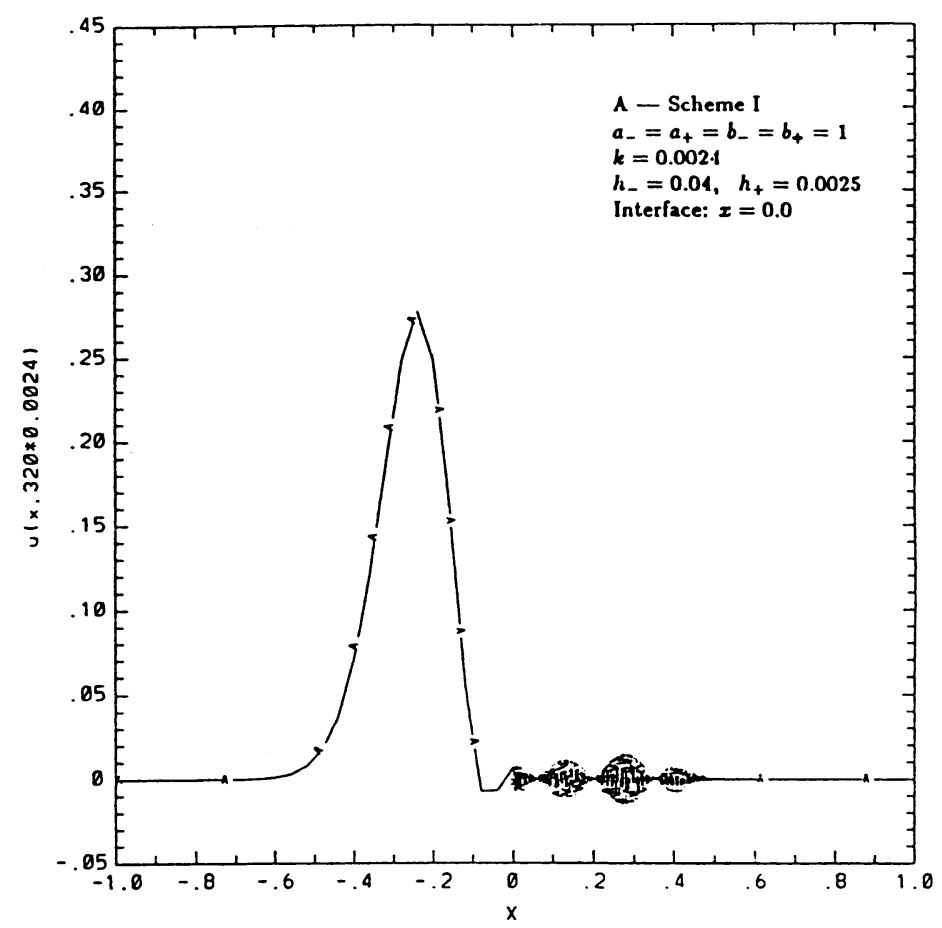

Figure 9 


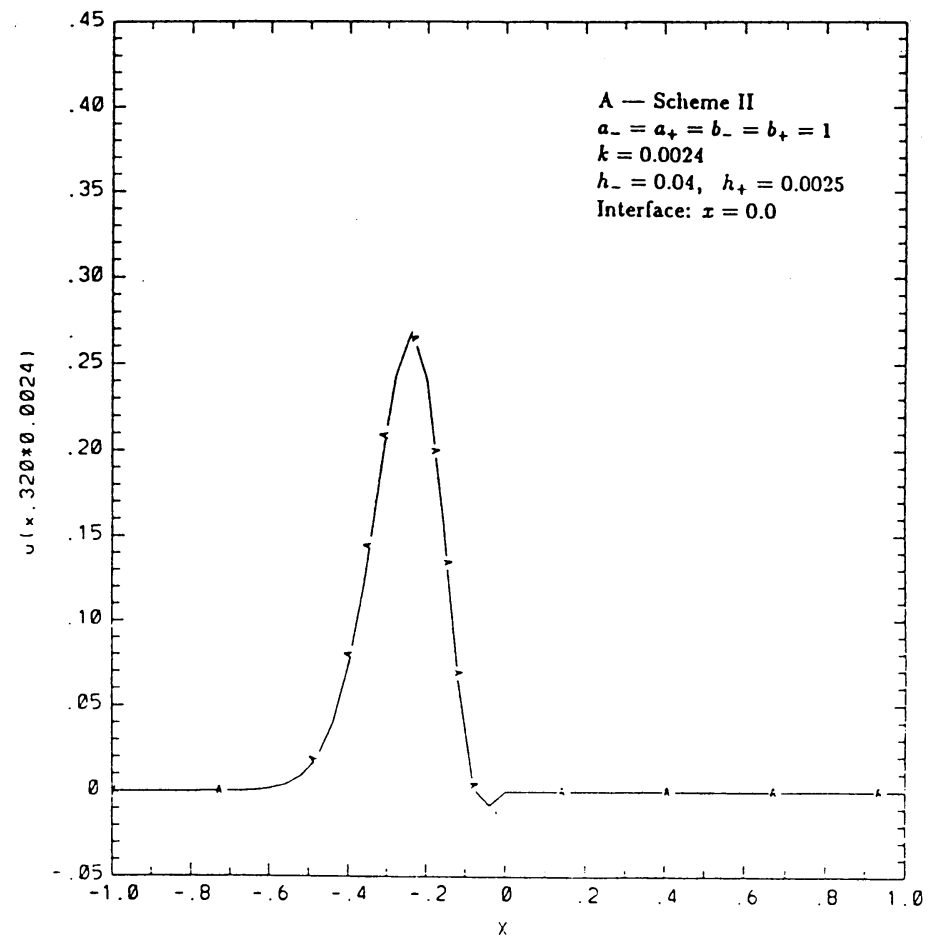

Figure 10

\section{BIBLIOGRAPHY}

1. M. Berger, Stability of interfaces with mesh refinement, Math. Comp. 45 (1985), 301-318.

2. J. Oliger, Hybrid difference methods for the initial boundary-value problem for hyperbolic equations, Math. Comp. 30 (1976), 724-738.

3. D. L. Brown, $A$ note on the numerical solution of the wave equation with piecewise smooth coefficients, Math. Comp. 42 (1984), 369-391.

4. G. Browning, H.-O. Kreiss, and J. Oliger, Mesh refinement, Math. Comp. 27 (1973), 29-39.

5. M. Ciment, Stable difference schemes with uneven mesh spacings, Math. Comp. 25 (1971), 219-227.

6. M. de Moura, Variable grids for finite difference schemes in numerical weather prediction, Pontificia Universidade Catolica de Rio de Janeiro, 1987.

7. B. Gustafsson, H.-O. Kreiss, and A. Sundström, Stability theory of difference approximations for mixed initial boundary value problems. II, Math. Comp. 26 (1972), 649-686.

8. G. Starius, On composite mesh difference methods for hyperbolic differential equations, Numer. Math. 35 (1980), 241-255.

9. A. Sundström, Efficient numerical methods for solving wave propagation equations for nonhomogeneous media, Stockholm Report, 1974.

10. L. Trefethen, Stability of finite difference models containing two boundaries or interfaces, Math. Comp. 45 (1985), 279-300.

11. __ Instability of difference methods for hyperbolic initial boundary value problems, Comm. Pure Appl. Math. 37 (1984), 329-367. 
12. L. Trefethen, Wave propagation and stability for finite difference schemes, Ph.D. Dissertation, Department of Computer Science, Stanford University, 1982.

13. R. Vichnevetsky, Wave propagation analysis of difference schemes for hyperbolic equations: a review, Internat. J. Numer. Methods Fluids 7 (1987), 409-452.

Department of Mathematics, University of Wyoming, LaRAmie, Wyoming 82071

Department of Mathematics, James Madison University, Harrisonburg, Virginia 22801

E-mail address: ewing@lode.uwyo.edu 